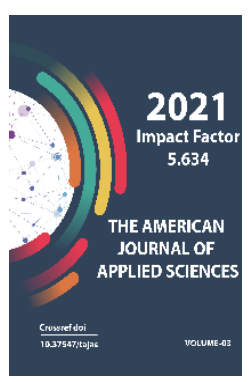

\title{
Characteristic Orbits Of Charged Particles Around Charged Black Holes
}

\author{
Xudoyberdiyeva Malika Karomat Qizi \\ (2-Course Bacis Doctoral Student) National University Of Uzbekistan, Tashkent 100174, \\ Uzbekistan
}

\section{Copyright: Original content from this work may be used under the terms of the creative commons attributes 4.0 licence.}

\section{ABSTRACT}

We have considered Reissner-Nordstr" om (RN) charged nonrotating black hole (BH).We have studied motion of charged particles around charged RN BH. It was found out that there are two boundary conditions for specific angular momentum of stable circular orbits corresponding to: innermost stable circular orbits (ISCO) and outermost stable circular orbits (OSCO) and accretion disk is originated between these two orbits. It was obtained the upper and lower limits for the value of particle's charge which may exist in the accretion disk matter around the extreme charged Reissner Nordstr"om black hole.

\section{KEYWORDS}

Black holes, Kerr-Taub-NUT, Blandford-Znajek mechanism ,Charged particle

\section{INTRODUCTION}

It is obvious properties of the spacetime can be studied considering the particle motion. Charged particle motion around ReissnerNordstr" om black hole has been studied in [1, 2].From astrophysical point of view it is interesting to study the charged particles motion around charged black holes and black holes in external magnetic field. Recently, the motion of charged [3, 4], magnetized and spinning particles around black holes with 
different parameters in an external asymptotically uniform magnetic field in various theories of gravity have been studied.

\section{LITERATURE REVIEW}

[4] A. A. Abdujabbarov, B. J. Ahmedov, and N. B. Jurayeva. Charged-particle motion around a rotating non-Kerr black hole immersed in a uniform magnetic field. Phys. Rev. D., 87(6):064042, March 2013.

[5] J. R. Rayimbaev. Magnetized particle motion around non-Schwarzschild black hole immersed in an external uniform magnetic field. Astrophys Space Sc, 361:288, September 2016

This research is supported by Grants No. VA-FAF-2-008, No. MRB-AN-2019-29 and No.YFAFtech-2018-8 of the Uzbekistan Ministry for Innovational Development, and by the Abdus Salam International Centre for Theoretical Physics through Grant No. OEA-NT-01.

\section{RESEARCH METHODOLOGY}

In this work, we have studied circular motion of charged particles around Reissner-Nordstr"om black hole and the following main results are obtained. It was shown that the critical radius of circular orbits depends on particle charge. Upper and lower limits for the value of charged particle at circular orbits for the given

value of RN black hole charge have been found

\section{ANALYSIS AND RESULTS}

The geometry of the spacetime around electrically and magnetically charged $\mathrm{RN} \mathrm{BH}$ in spherical coordinates $\left(x^{\alpha}=\{t, r, \theta, \phi\}\right)$ is given in the following form $d s^{2}=S(r)\left[-f d t^{2}+f^{-1} d r^{2}+r^{2}\left(d \theta^{2}+\right.\right.$ $\left.\sin ^{2} d \phi^{2}\right]$

(1)

with the following gravitational metric function

$f=1-\frac{2 M}{r}+\frac{Q^{2}+P^{2}}{r^{2}}$

and associated with the four vector potential of the electromagnetic field around the electrically charged $\mathrm{BH}$

$A_{\alpha}=\frac{Q}{r}\{1,0,0,0\}$

where $M$ is the total mass of $B H, Q$ and $P$ is the total electric and magnetic charge, respectively.Here we will provide the study of charged particle motion around charged black hole, supposing $m$ is particle mass and $e$ is electric charge of the particle. The Lagrangian for the charged particle in the electromagnetic field has the following form

$\mathcal{L}=m g_{\mu \nu} u^{\mu} u^{v}+e u^{\mu} A_{\mu}$

The conserved quantities \{ the energy and the angular momentum can be found by

$g_{t t} \dot{t}+q A_{t}=\varepsilon$

$g_{\phi \phi} \dot{\phi}=\mathcal{L}$

where $\varepsilon=E / m$ and $\mathcal{L}=L / m$ are the specific energy and angular momentum of the particle, respectively, $q=e /(m c)$ is the specific electric charge of the particle with mass $m$ and electric charge $e$, related to the observer at infinity. The equation of motion for charged particles 
with the Lagrangian (4) can be found using the Euler-Lagrange equation [5], so we have

$u^{\mu} \nabla u^{v}=q F_{\sigma}^{v} u^{\sigma}$

where $F_{\sigma}^{v}=g^{\mu v} F_{\mu \sigma}$ and $F_{\mu \sigma}=A_{\sigma, \mu}-A_{\mu, \sigma}$ is the electromagnetic field tensor. Using the equations (5-7) one may easily find the equation of motion of the charged particles in the equatorial plane $(\theta=\pi / 2)$ in the following form

$$
\begin{array}{r}
\dot{t}=\frac{1}{f}\left(\mathcal{E}-\frac{Q q}{r}\right) \\
\dot{r}^{2}=\left(\mathcal{E}-\frac{Q q}{r}\right)^{2}-f\left(1+\frac{\mathcal{L}^{2}}{r^{2}}\right) \\
\dot{\phi}=\frac{\mathcal{L}}{r^{2}}
\end{array}
$$

In this section we will study the stable circular orbits using following standard conditions

$V_{\text {eff }}=\varepsilon, \quad V_{\text {eff }}=0$,

in the equatorial plane the circular orbits can be stable for the critical value of angular momentum $L_{c r}$ which is the solution of the equation $V_{e f f^{\prime}}=0$ and have the following form

$\mathcal{L}_{ \pm}^{2}=\frac{1}{2\left(r(r-3 M)+2 Q^{2}\right)^{2}}\left[Q^{2} r^{3}\left(\left(q^{2}-2\right) r-\right.\right.$ $\left.2 M\left(q^{2}-5\right)\right)+2 M r^{4}(r-3 M)+\left(q^{2}-\right.$
4) $Q^{4} r^{2} \pm q Q r^{2}(r(r-2 M)+$

$\left.\left.Q^{2}\right) \sqrt{4 r(r-3 M)+\left(q^{2}+8\right) Q^{2}}\right]$

Now we will analyze the solution (11) and look for the condition where both $\mathcal{L}_{ \pm}^{2}$ are real. For this we require the expression inside the square root to be non-negative:

$4 r(r-3 M)+\left(q^{2}+8\right) Q^{2} \leq 0$

From the condition (12) one may get the lower limit for radius of the circular orbit of particle when $L$ is still real.

$$
r_{\text {crit }}=\frac{3 M}{2}\left(1+\sqrt{1-\frac{\left(q^{2}+8\right)}{9} \frac{Q^{2}}{M^{2}}}\right.
$$

Expression indicates the allowed value of the charge of the test particle required for circular stable orbits. Figure 1 illustrates the dependence of critical radius of circular orbits from the electric charge of the $\mathrm{BH} Q$ and the particle $q$. One can see that the critical radius decreases with the increase of the value of $Q$. From the Figure 1 one can also see that for the neutral test particle we get $r=3 M$ (the Scwarzchild case). It can be also seen from the figure that for the large values of $Q$ and $q$, the critical radius decreases very fast, depending both black hole and particles charge. The critical radius decreases with the increase of the black hole chargeand reaches the value of $2 M$ for $Q=1$ for neutral particl 


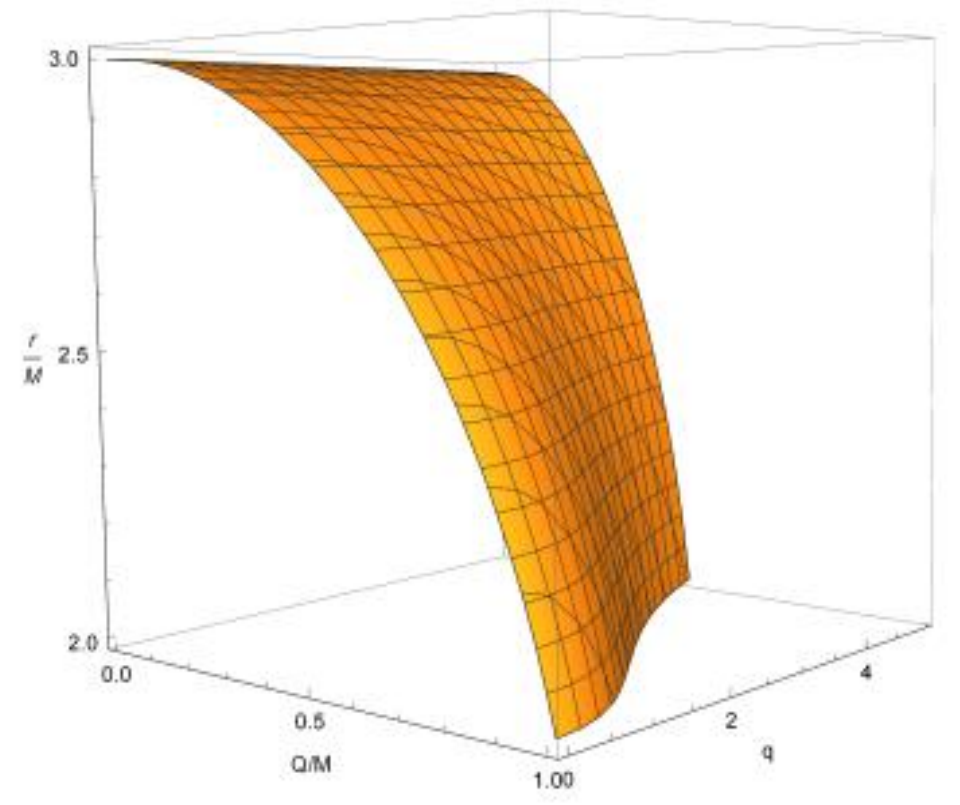

Figure 1: 3D plot for the dependence of critical radius of circular orbits on the charge of $B H Q$ and particle $q$.


Figure 2: ISCO and OSCO radius as a function of black hole charge for negatively charged particles.

Figure 2 illustrates the dependence of ISCO and OSCO radius on black charge for negatively charged particles. One can see that in both case $q>1$ and $q<1$ ISCO radius decreases with the increase of the value of black hole charge.
The behavior of the radius of OSCO differs from the case of positive charge: for all negative charge of particles the radius of OSCO increases with the increase of the module of the charge. 

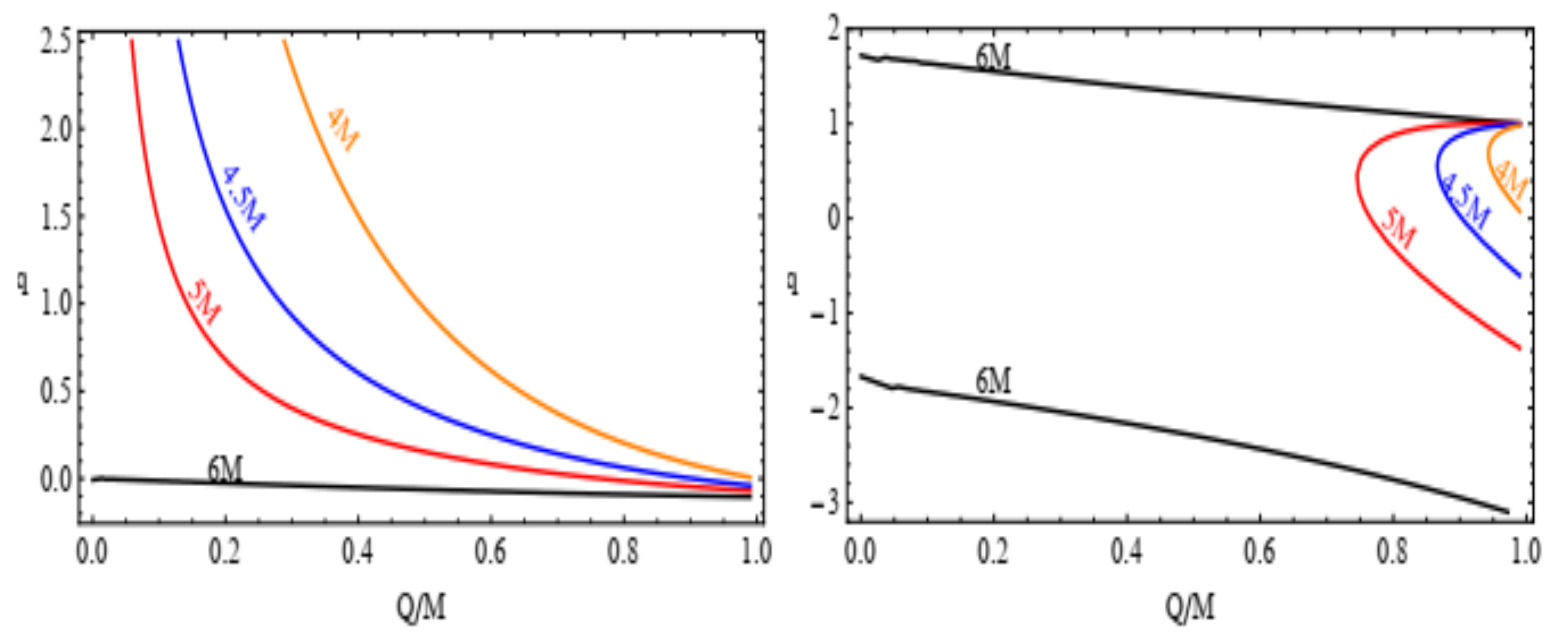

Figure 3: $q-Q$ diagram for different values of ISCO (left panel) and OSCO (right panel) radius of a charged particle

Now we study thedistance between ISCO and OSCO, which corresponds to the size of accretion disk $\Delta r=r_{\text {osco }}-r_{\text {isco }}$

In Figure 3 we present the relation between the particle's and the black hole's charges for the fixed values of ISCO and OSCO. One can see from the diagram that OSCO may not exist below the radius $6 \mathrm{M}$ from the central object for negatively charged particles and for positively charged particles with value more than $q=2$. One can also see from the right panel of the .Figure 3 OSCO radius (at the range $6 M \leq$ $\left.r_{\text {osco }} \leq 4 M\right)$ can be the same for different charged particles $(2 \leq q \leq-3)$ for the fixed value of black hole charge.

\section{CONCLUSIONS}

It was found that there are two critical values for the specific angular momentum for charged particles. One of them corresponds to the lower boundary and the other to the upper boundary of the stable circular orbits. It was shown that OSCO radius of positively charged particles increases with the increase of $Q$ for $q>1$ and it decreases with the increase of $Q$ fr $q<1$. However, OSCO radius for negatively charged particles increases with the increase of the black hole charge $Q$.

\section{REFERENCES}

1. D. Pugliese, H. Quevedo, and R. Ruffini. Circular motion in Reissner-Nordstrn"om spacetime. ArXiv e-prints, March 2010.

2. D. Pugliese, H. Quevedo, and R. Ruffini. Motion of charged test particles in Reissner-Nordstr"om spacetime. Phys. Rev. D, 83(10):104052, May 2011.

3. Bakhtiyor Narzilloev, Ahmadjon Abdujabbarov, Cosimo Bambi, and Bobomurat Ahmedov. Charged particle motion around a quasi-Kerr compact object immersed in an external magnetic field. Phys. Rev. D, 99(10):104009, May 2019

4. A. A. Abdujabbarov, B. J. Ahmedov, and N. B. Jurayeva. Charged-particle motion around a rotating non-Kerr black hole immersed in a uniform magnetic field. Phys. Rev. D., 87(6):064042, March 2013. 
The American Journal of Applied sciences (ISSN - 2689-0992)

Published: April 28, 2021 | Pages: 78-83

PACT FACTOR

Doi: https://doi.org/10.37547/tajas/Volume03Issue04-10

2021:5. 634

5. J. R. Rayimbaev. Magnetized particle motion around non-Schwarzschild black hole immersed in an external uniform magnetic field. Astrophys Space Sc, 361:288, September 2016. 